\title{
Estudio de Desarrollo de Niños Considerados de Alto Riesgo Neurológico en el Periodo Neonatal
}

\author{
Drs. J.P. Beca*, C. Román*, L. Zubaty**, J. Abourbih"**.
}

\begin{abstract}
I follow-up study was gerfornted in a group of 37 children who were considered tir be "neurslugiral high riak" patients during their neonargl period, A contrul grump of $24 \mathrm{~s}$ bildren was mually studied. Development was evaluated in

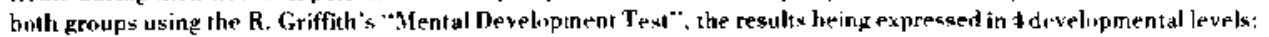
normal. mild. moderate and proftund retardation.

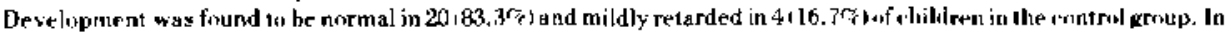

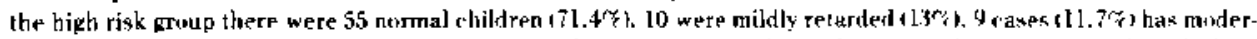
ate. and $31,3,0 \%$ bad profound developmental retardation. Resulss in the study gramp did not differ sigrsificanıly from those in the enutrol group.

Develupmental levels in (tbildren whose birth weight was under I 501 gr 456 sases), with low hirh weight for their ges-

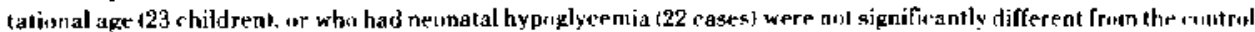
group. However development was fuund to be normal in $89.7 \%$ of children whose fatmilies had a hixh or intermmliate

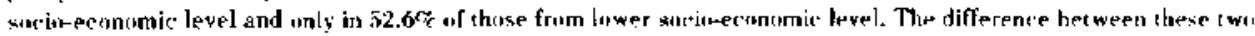
sub-proups was siguifirant.
\end{abstract}

Durante los últimos años la atención del recién nacido ha variado sustancialmente poniéndose énfasis tanto en lograr disminuir la mortalidad neonatal y perinatal, como en reducir el riesgo de adquirir secuelas neurológicas o del desarrollo psicomotor. El esfuerzo por prevenir y tratar adecuadamente la asfixia perinatal. los trastornos respiratorios del recién nacido, la hiperbilirrubinemia, la hipoblicemia neonatal. los trastornos de coagulación, las infecciones. etc.. han levado en la mayoría de los centros de atención perinatal a una disminución simultánea de las tasas de mortalidad y del número de niños con secuelas neurológicas. En nuestro medio estamos igualmente viviendo la puesta en marcha de un "Programa de prevención del daño neurológico y disminución de la mortalidad neonatal". ${ }^{1}$ Estos objetivos se alcanzarán mediante las acciones obstétricas y neonatológicas debidamente coordinadas entre sí. Entre las premisas ofundamentos de este programa se considera una estimación de que en Chile se producen alrededor de 3.200 casos de secuelas neurológicas graves al año. Si bien esta estimación es razo-

\footnotetext{
•Centro de Herematuros, Hospital L. Calvo Mackenna.

"Vylunaria Cuerpo de Paz iEtstados Unidos]

***Estudiante de Psicolngia. Unjuersidad Catálica de Chile
}

nable, no existe un estudio nacional que aporte datos seguros o una orientación precisa sobre la magnitud del problema, o sobre el pronóstico de desarrollo de los recién nacidos patológicos.

Estudios extranjeros muestran un mejoramiento progresivo del pronóstico neurolúgico o de desarollo de los recién nacidos patológicos o de menor peso de nacimiento. El mejor pronóstico se ha relacionado estrechamente con la mejor atención médica prenatal, del parto y del período neonatal. Los resultados han ido mejorando en la medida en que se han ido introduciendo técnicas cada vez más complejas en los centros de alto riesgo obstétrico y de cuidado intensivo neonatal. Como la introducción de estas técnicas se realiza con mayor velocidad que la necesaria para realizar estudios de seguimiento de desarrollo a largo plazo, resulta imposible predecir el futuro de niños nacidos en esta época sobre la base de estudios realtzados en niños nacidos varios años antes. Esta limitación es aún mucho mayor si los seguimientos han sido realizados en casuisticas que difieren entre sí y en países o en centros con diferentes condiciones de atención médica.

Con el objeto de obtener una orientación preliminar sobre el pronóstico de desarrollo de recién nacidos patológicos y/o de muy bajo peso al nacer, 
atendidos en nuestro medio, realizamos la presente investigación. Se llevó a cabo un estudio de seguimiento de un grupo de recién nacidos arbitrariamente definidos como de "alto riesgo neurológieo", hospitalizados en el Centro de Prematuros y del Hospi1al L. Calvo Mackenna entre 1973 y 1975. Se evaluó el desarrollo de una edad fluctuante entre 2 y 4 años. Para estimar la validez del instrumento aplicado fue necesario aplicar previamente el test de desarrollo a un grupo control de niños sin antecedentes de patologia perimatal.

\section{MATERIAL}

El grupo de estudio se estableció citando por carta a todos los niños dados de alta del Centro de Prematuros entre 1973 y 1975 que hubieran presentado por lo menos una de las condiciones siguientes: peso de nacimiento igual o inferior a 1.500 grs., hipoglicemia neonatal, desnutrición fetal, hemorragia intracraneana de tipo subaracnoídeo, o hiperbilirrubinemia sobre $15 \mathrm{mg} \%$. No se incluyó en el estudio a nin̄os con otros factores de riesgo neurológico por no disponerse de antecedentes precisos. Fue posible completar el estudio en 56 niños cuyo peso de nacimiento fue 1.500 grs. o menos, 22 niños que tuvieron hipoglicemia neonatal, 23 casos que presentaron desnutrición fetal, 6 casos de hemorragia subaracnoídea y 16 casos de hiperbilirrubinemia. Los casos de hiperbilirrubinemia que no estaban incluidos además en otros grupos fueron posteriormente excluidos del estudjo por constituir un grupo beterogéneo de niños con mínimo riesgo neurológico. Se constituyó así un grupo de estudio de 77 recién nacidos que se analizan en conjunto y por separado según la patología neonatal.

El grupo control comprendió 24 niños preescolares asistentes a 3 jardines infantiles de distintos niveles sucioeconúmicos. en los cuales se pudo obtener el antecedente de haber tenido un período perinatal y de lactante sin patología de importancia. Se incluyeron en el grupo control a 7 niños de 2 años. 9 niños de 3 años y a 8 niños de 4 años.

\section{METODO}

Tanto a los niños del grupo control como a los del grupo de estudio se les aplicó la "Escala de Desarrollo Mental de Ruth Griffiths"'(2). El test evalúa globalmente 6 áreas o subescalas que son: motora gruesa, motora fina, coordinación viso-manual, personal social, audición-lenguaje y razonamiento práctico.
Los resultados del test se expresaron en 4 niveles de desarrollo de acuerdo a las definiciones siguientes:

- desarrollo normal; rendimiento normal o superior en todas las áreas.

- retraso leve del desarrollo: ligera desviación del promedio normal de capacidad en una o más áreas. El problema puede ser superado o aliviado con estimulación adecuada y apoyo pedagógico.

- retraso moderado del desarrollo: desviación notable del promedio normal de capacidad en una o más áreas. Los problemas pueden ser parcialmente aliviados mediante educación especial.

- retraso profundo del desarrollo: rendimiento muy por debajo del promedio normal en varias áreas. mínima participación en el medio ambiental. Estos niños pueden ser educados para alcanzar habilidades básicas de cuidado personal.

En 20 casos del grupo de estudio los resultados del test de desarrollo fueron dudosos por haberse detectado problemas atribuibles a factores de tipo circunstancial o situacional. En estos casos el mismo examinador repitió el test 6 a 10 meses después, encontrándose muy poca variación en relación a los resultados de la primera evaluación

Además de analizarse los niveles de desarrollo obtenidos en los niños del grupo control y del grupo de alto riesgo neurológico, se analizaron los resultados de los distintos subgrupos por separado. según peso de nacimiento y patologia perinatal. Se detallan los antecedentes clinicos perinatales y de la edad de lactante de los casos que presentaron alteraciones moderadas o profundas del desarrollo.

Se relacionaron los niveles de desarrollo de los niños del grupo de alto riesgo neurológico con el nivel socioeconómico familiar. Este se estableció por una encuesta básica del nivel económica, educacional y habitacional realizada al aplicar el test.

El análisis estadístico se realizó empleando la prueba de $\mathrm{X}^{2}$. Se consideró significativo $\mathrm{p} \leqq 0.05$.

\section{RESULTADOS}

El test de Griffiths aplicado a $\operatorname{los} 24$ niños del grupo control de 2 a 4 años mostró 4 casos (16.7\%) de niños con retraso leve del desarrollo (Tabla N. ${ }^{\circ}$ 1). El retraso del desarrollo fue en estos casos predominantemente en las áreas de lenguaje y de coordinación viso-manual. 
Nivel de Desarrollo de 24 niños de 2 a 4 años sin antecedentes de patología perinatal.

\begin{tabular}{lcc}
\hline Desarrollo & N. & \multicolumn{1}{c}{ sc } \\
\hline Normal & 20 & 83.3 \\
Retraso leve & 4 & 16.7 \\
Retraso moderado & - & - \\
Retraso profundo & - & - \\
\hline Total & 24 & 100 \\
\hline
\end{tabular}

En el total de 77 niños considerados de alto riesgo neurológico hubo 55 niños con desarrollo normal (71.4\%), 10 casos con retraso leve $(13 \%), 9$ con retraso moderado (11.7\%), y 3 niños con retraso profundo del desarrollo (3.9\%). (Ver Tabla N. ${ }^{\circ}$ 2). La comparación del nivel de desarrollo del grupo de niños con alto riesgo neurológico con el del grupo control no mostró diferencia significativa ( $p>0.05)$.

\section{TABLA N. ${ }^{\circ} 2$}

Nivel de Desarrollo a los 2 a 4 años de niños considerados de "allo riesgo neurológico" en el período neonatal

\begin{tabular}{lrr}
\hline \multicolumn{1}{c}{ Desarrollo } & N. & $\%$ \\
\hline Normal & 55 & 71.4 \\
Retraso leve & 10 & 13.0 \\
Retraso moderado & 9 & 11.7 \\
Retraso profundo & 3 & 3.9 \\
\hline Total & 77 & 100 \\
\hline
\end{tabular}

$(p=>0.05)$

Entre los 77 niños del grupo de estudio hubo 56 con peso igual o inferior a $1.500 \mathrm{grs}$. al nacer. La evaluación del desarrollo a los 2 a 4 años demostró que 38 de ellos (67.9\%) tenían desarrollo normal, 8 tenían retrasso leve (14.\%), 7 tenían retraso moderado (12.5\%) y 3 niños tenian retraso profundo del desarrollo (5.3\%). (Tabla N. ${ }^{0} 3$ ). La comparación de los niveles de desarrollo de los niños con peso de nacimiento inferior a 1.500 grs. con los del grupo control no mostró diferencia significativa $(p>0.05$ ).

El análisis de los resultados obtenidos en los njños con menor peso de nacimiento mostró que hubo en la serie 21 niños con peso igual o inferior a 1300 grs. Entre ellos no hubo casos con retraso moderado ni profundo del desarrollo. y sólo 2 de los 21 casos (9.5\%) tenian retraso leve, resultado que no difiere del grupo control.
Nivel de Desarrollo a los 2 a 4 años de niños con peso de nacimiento inferior a $1.501 \mathrm{grs}$.

\begin{tabular}{lrr}
\hline \multicolumn{1}{c}{ Desarrollo } & $\mathrm{N}{ }^{\circ}$ & $\%$ \\
\hline Normal & 39 & 67.9 \\
Retraso leve & $\mathrm{g}$ & 14.3 \\
Retraso moderado & 7 & 12.5 \\
Retraso profundo & 3 & 5.3 \\
\hline Total & 56 & 100 \\
\hline
\end{tabular}

$(p=>0.05)$

Los niveles de desarrollo de los 22 niños con antecedentes de haber presentado hipoglicemia en los primeros días de vida muestran que: (Tabla $\mathrm{N}^{\circ}{ }^{\circ}$ 4.) De $\operatorname{los} 22$ casos. 17 tenian desarroljo normal (77.3\%): 2 tenían retraso leve $(9.1 \%) ; 3$ niños tenían retraso moderado (13.6\%). y ninguno tenía retraso profundo. Los resultados de este grupo tampoco difieren significativamente del grupo control ( $\mathrm{p}>0.05$ ). En los 3 casos con retraso moderado había, además de la hipoglicemia, otros factores de riesgo: enfermedad hemolítica por Rh con hiperbilir rubinemia hasta de $17 \mathrm{mg} \%$. hipoxia perinatal grave, y poligiubulia respectivamente. (Ver Tabla $N^{\circ}{ }^{\circ} 6$, casos $4,5 y$ 6.) Los 2 casos de esle grupo con retraso leve del desarrollo tuvieron hipoglicemia sintomática, y también tenían otros factores de riesgo: desnutrición fetal e hipoxia neonatal con SDRI grave respectivamente.

TABLA N. ${ }^{\circ} 4$

Vivel de Desarrollo a los 2 a 4 años de niños con antecedentes de Hipoglicemia Neonatal

\begin{tabular}{lrc}
\hline Desarnollo & N. & $\%$ \\
\hline Normal & 17 & 77.3 \\
Retraso leve & 2 & 9.1 \\
Retraso moderado & 3 & 13.6 \\
Retraso profundo & - & - \\
\hline Total & 22 & 100 \\
\hline
\end{tabular}

$(\mathrm{p}=>0.05)$

Entre los 23 niños con antecedentes de desnutrición fetal (Tabla N. ${ }^{\circ} 5$ ) huto 15 niños con desarrollo normal (65.2\%), 4 con retraso leve (17.4\%), 1 caso con retraso moderado $(4.4 \%)$, y 3 casos con retrasn profundo de] desarrollo (13\%). Aun cuando el 
número de niños con desarrollo normal en este grupo es menor que en los demás subgrupos analizados. la diferencia con el grupo control tampoco es significativa. Los 3 casos con retraso profundo tenian patulugía grave asociada, que se analiza măs adelante. El caso con retraso moderado del desarrollo tiene enanismo y signos clínicos de genopatía que no se han podido precisar por rechazo de los padres al estudio genético. Los 4 casos de niños desnutridos fetales que presentaban retraso leve del desarrollo en el momento del estudio también tenian otros antecedentes de patología que puede ímplicar riesgo neurológico: SRDI. hipoglicemia, atresia esofágica, e hipoxia neonatal. respectivamente.

TABLA N. ${ }^{\circ} 5$

Nivel de Desarrollo a los 2 a 4 años de niños con peso de nacimiento bajo para su edad gestacional

\begin{tabular}{lrr}
\hline Desarrollo & $\mathrm{N}^{\circ}$ & $\%$ \\
\hline Normal & 15 & 65.2 \\
Retraso leve & 4 & 17.4 \\
Retraso moderado & 1 & 4.4 \\
Retraso profundo & 3 & 13.0 \\
\hline Total & 23 & 100 \\
\hline
\end{tabular}

(p $=>0.05)$

Hubo 6 casos en los cuales se demostró la existencia de hemorragia subaracnoídea mediante la comprobación de líquido céfalo-raquídeo hemorrágico en por lo menos 2 punciones lumbares. Entre los antecedentes clínicos de estos niños se destaca que 4 de ellos pesaron menos de $1500 \mathrm{grs}$. al nacer, 3 de ellos fueron recambiados por hiperbilirrubinemia, y 2 de ellos tuvieron hipoglicemia asociada. El estudio de desarrollo se realizó a los 2 años en 3 casos y a los 3 años de edad en los otros tres. Los 6 niños tuvieron test de desarrollo normal.

En las Tablas N." 6 y 7 aparecen los antecedentes clínicos más importantes de los niños que presentaban retrasos moderado o profundo del desarrollo. Se resumen en las Tablas sólo los datos clínicos que pueden tener relación causal con los retrasos respectivos.

Entre los antecedentes de los 9 niños con retraso moderado del desarrollo llama la atenciśn que 3 de ellos tenían malformaciones congénitas diversas, 3 niños uuvjeron hipoglicemia, y 2 casos tuvieron poliglobulia (Tabla N. ${ }^{\circ}$ ). Los casos 7 y 8 tenían problemas sociales graves que pueden influir negativamente en el desarrollo. En el caso $N 0^{\circ} 9$ no se demostraron antecedentes clínicos que pudieran alterar el desarrollo.

\section{TABLA N.*6}

Antecedentes clínicos de niños con retraso moderado del desarrollo

\begin{tabular}{|c|c|}
\hline Caso N. ${ }^{\circ}$ & Antecedentes clínicos \\
\hline 1 & $\begin{array}{l}\text { RN Pret } 32 \text { sem AEG, } 1500 \mathrm{~g} . \\
\text { SDRI, sepsis } \\
\text { Genopatía, enanismo }\end{array}$ \\
\hline 2 & $\begin{array}{l}\text { RN Pret. } 31 \mathrm{sem} \text { AEG, } 1390 \text { g. Gemelo II } \\
\text { Malformaciones múltiples de extremidades } \\
\text { Sepsis neonetal }\end{array}$ \\
\hline 3 & $\begin{array}{l}\text { RN Pret. } 32 \text { sem AEG, } 1400 \text { g., Gemelo I } \\
\text { Cataratas congénitas }\end{array}$ \\
\hline 4 & $\begin{array}{l}\text { RN Pret. } 30 \text { sem AEG, I } 40 \mathrm{~g} \text {. } \\
\text { Hipoglicemia sinlomática } \\
\text { lctericia por lncompst. Rh (Bil. } 17 \mathrm{mg} \%\end{array}$ \\
\hline 5 & $\begin{array}{l}\text { RN Pret } 34 \text { sem AEG, } 1800 \mathrm{~g} . \\
\text { Hipoxia perinatal grave } \\
\text { Hipoglicemia sintomâtica } \\
\text { Poliglobulia }\end{array}$ \\
\hline 6 & $\begin{array}{l}\text { RN Pret. } 34 \text { sem AEG. } 1820 \mathrm{~g} . \\
\text { Hipoglicemia asintomática } \\
\text { Poliglobulia }\end{array}$ \\
\hline 7 & $\begin{array}{l}\text { RN Pret. } 33 \text { sem AEG. } 1500 \mathrm{~g} \text {. } \\
\text { Desnutrición del lactante } \\
\text { Problema social grave }\end{array}$ \\
\hline 8 & $\begin{array}{l}\text { RN Pret. } 36 \text { gem PEG, } 1500 \mathrm{~g} \text {. } \\
\text { Problema social grave }\end{array}$ \\
\hline 9 & RN Pret. $32 \mathrm{gem}$ AEG, $1400 \mathrm{z}$. \\
\hline
\end{tabular}

Los antecedentes clinicos de los 3 niños con retraso profundo del desarrollo (Tabla N. ${ }^{\circ}$ ) muestran en el caso $\mathrm{N}^{\circ} \mathrm{I}$ un Síndrome 5 p (-) con microcefalia congénita extrema; - los otros 2 niños son hermanos gemelos, desnutridos fetales, con desnutrición moderada posterior, y graves problemas sociales en la familia. El caso N. ${ }^{\circ}$ tuvo infecciones urinarias a repetición sin estudio urológico, y el gemelo II (caso N. ${ }^{\circ} 3$ ) tuvo a los 8 días de edad una diarrea aguda con deshidratación e hipematremia.

El anălisis del nivel de desarrollo de los niños considerados de alto riesgo neurológico en relación al nivel socio-económico familiar aparece en la Tabla N. ${ }^{\circ} 8$. Hubo 11 casos de niños pertenecientes a familias de nivel socioeconómico alto y 28 de nivel 
medio que se analizan en conjunto. Entre ellos hubo 35 niños normales $(89.7 \%), 3$ con retraso leve $(7.7 \%)$, l caso con retraso moderado ( $2.6 \%)$, y ninguno con retraso profundo del desarrollo. Entre los 38 niños pertenecientes a familias de nivel socioeconómico bajo hubo 20 niños normales $(52.6 \%) .7$ casos con retrasu leve ( $18.4 \%), 8$ casos con retraso moderado (21.1\%) y 3 casos con retraso profundo del desarrollo $(7.9 \%)$. La diferencia entre ambos grupos socioeconómicos es significativo $(\mathrm{p}<0.01)$.

\section{TABLA N: 7}

Antecedentes clínicos de niños con retrago profundo del desarrollo

\begin{tabular}{|c|c|}
\hline Caso $N^{\circ}$ & Antecedentes clínicos \\
\hline 1 & $\begin{array}{l}\text { RNT } 40 \text { sem. PEG, } 1440 \mathrm{~g} \\
\text { Sindrome Sp (-) } \\
\text { Mierocefalia congénita }\end{array}$ \\
\hline 2 & $\begin{array}{l}\text { RN Pret } 36 \text { sem PEG. I } 470 \text { g. . Gemelo I } \\
\text { Infecciones urinarias a repetición } \\
\text { Desnutrición del lactante } \\
\text { Problems social grave }\end{array}$ \\
\hline 3 & $\begin{array}{l}\text { RN Pret. } 36 \text { sem PEG, } 1420 \mathrm{~g} . \text {. Gemelo } 1 \\
\text { Diarrea aguda e hipernatremia (8 dias) } \\
\text { Desnutrición del lactante } \\
\text { Problema social grave }\end{array}$ \\
\hline
\end{tabular}

TABLA N." 8

Nivel de Desarrollo de niños de "alto riesgo neurológico" según su nivel socioecanómico familiar

Nivel socioeconómico

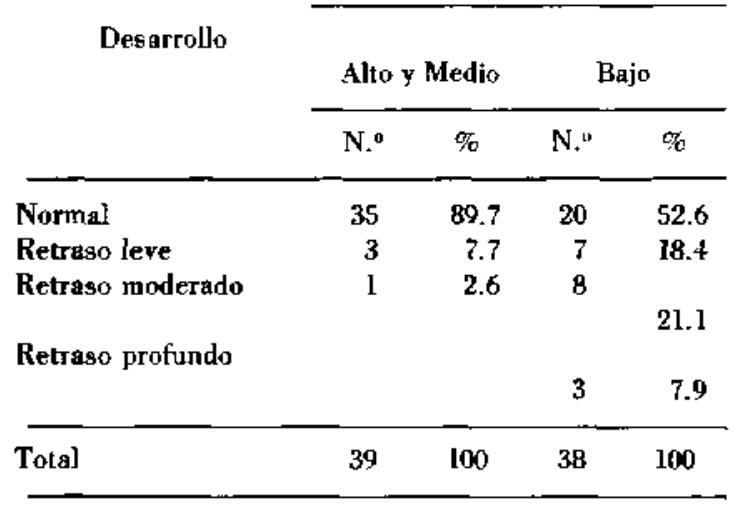

$\{\mathbf{p}=<0.01\}$

\section{DISCLSION}

El análisis de lns resultados de la presente investigación. como el de todo estudio de seguimiento del desarrollo o de pronóstico neurolígico, debe ser extremadamente cuidadoso. Los resultados deben entenderse e interpretarse como válidos sólo para el material escogido para este estudio, es decir, atendidos en un Centro de referencia, con las condicion nes de trabajo propias del Centro de Prematuros del Hospitàl L. Calvo Mackenna entre 1973 y 1975. Los resultados pueden ser diferentes para otra poblacińn. atendida en otros centros. con otras condiciones de trabajo y en épocas diferentes.

E] instrumento escogido para evaluar globalmente el desarrollo de niños entre 2 y 4 años de edad fue el Test de Ruth Griffiths. Este evalúa globalmente las áreas más arriba definidas, ha șido ampliamente empleado en el Reino Unido, y se ha aplicado en el Departamento de Neurología del Hospital L. Calvo Mackenna desde 1974. Si bien el Test no ha sido sometido en Chile a una estandarización estadística, su uso previo ha demustrado ser un instrumento aplicable en nuestro medio. Los resultados obtenidos en el grupo control de nuestra investigación son por lo demás los esperable en una población normal a $\operatorname{los} 2$ a 4 años de edad. No puede sin embargo descartarse la posibilidad de que otros tests que evalúen más especificamente ciertas áreas o funciones pudieran revelar otras diferencias entre el arupo de esludio y el grupo control.

El grupo control, constituido por 24 niños asistentes a 3 jardines infantiles diferentes. tiene valjdez. por cuanto está integrado por niños de distintos niveles socioeconómicos. Pero el número reducido del grupo control no permitió comparar el grupo de estudio con el grupo control dividido por niveles socioeconímicos, lo que hubiera dado mayor validez al análisis de esta importante variable. Por otra parte. podría esperarse que los niños del grupo control tuvieran mejores resultados en el Test de Griffiths por la estimulación que reciben en los respectivos jardines infantiles.

Los resultados globales del grupo arbitrariamente definido como de "alts riesgo neurológico" muesıran $71.4 \%$ de niños con desarrollo normal. $13 \%$ con retraso leve, $11.7 \%$ con retraso moderado. y $3.9 \%$ con retraso profundo. Estos niveles de desarrollo si bien difieren del grupo control, Jas diferencias no alc anzan significación estadística $(p>0.05$ ). Resultados de estudios de seguimiento en la literatura muestran efectos muy variados sezún la épora del estudio. los criterios de seguimiento, y el material 
estudiado. Así L. Lubcheneo en $1963^{3}$ da cifras de hasta $68 \%$ de secuelas en un seguimiento de 10 años, y en 1964 da porcentajes de secuelas entre 6 y $100 \%$,según edad gestacional y peso de nacimiento 4. I. Bjerre en $1975^{5}$ publica secuelas graves en $3.6 \%$ y leves o moderadas en $36.7 \%$ de los recién nacidos de bajo peso en su serie. P. A. Davies' demuestra una reducción de la incidencia de diplejia espástica en recién nacidos de menos de 1500 grs. al nacer de $10.3 \%$ en 1961 a $0 \%$ en 1970. K. E. Pape? muestra en 1978 similar mejoría del pronóstico comparando niños de 1000 grs. o menos nacidos en 1970 y 1974 respectivamente. La muestra de la presente invegtigación, si bien señala resultados similares. no es comparable con los estudios citados dadas las características de nuestro material y las distintas condiciones de trabajo.

Los 56 niños cuyo peso de nacimiento fue inferior a 1500 grs. tuvieron niveles de desarrollo similares al grupo total de estudio y no diferentes al grupo control. El análisis del desarrollo de aquellos con menor peso de nacimiento (1 300 grs. o menos) no permitió establecer relación entre peso de nacimiento y pronóstico de desarrollo ya que los resultados fueron aún mejores al grupo total. Este resultado apoya el concepto de que el peso de nacimiento por sí mismo no significa riesgo neurológico, dependiendo éste del conjunto de factores o complicaciones perinatales asociadas, como lo han señalado $\mathrm{L}$. Lubchenco ${ }^{8}$ y P. A. Davies 9 .

Los niños con antecedentes de hipoglicemia neonatal tampoco dífirieron del grupo control en su desarrollo. Aquellos niños de este grupo con retraso del desarrollo presentaban otras complicaciones asociadas, to que hace imposible establecer una relación causa-efecto entre hipoglicemia neonatal y secuelas neurológicas en esta casuística. Este resultado coneuerda con aquellos de Griffiths y Bryant 10, en cuanto a la dificultad de interpretar el rol de la hipoglicemia aislada de otras condiciones clinicas en la etiología de secuelas neurológicas.

Los resultados de niveles de desarrollo en niños con antedecentes de desnutrición fetal, aunque sin alcanzar diferencias significativas con el grupo control, son peores que los de los demás subgrupos analizados. Es precisamente en este grupo de niños donde se encentraron tres casos con retraso profundo del desarrollo, pudiendo atribuirse éste a una genopatía en un caso, y a la suma de desnutrición fetal, infecciones postnatales y problemas sociales en los otros dos (Tabla N. ${ }^{\circ}$ 7). Un caso de este grupo tuvo un retraso moderado del desarrollo atribuido a una genopatia (Tabla $N,{ }^{\circ} 6$ ), y los casos con retraso leve tenian además otros factores potencialmente causantes de secuelas. Estos hallazgos concuerdan con los de J. O. Commey y cols. I' quienes señalan a los recién nacidos con bajo peso para su edad gestacional como un grupo muy heterogéneo en el cual hay mayor frecuencia de alteraciones prenatales, intraparto y postnatales. Por esta razón, además de los riesgos propiamente nutricionales 12 13. 14 . los desnutridos fetales han sido siempre señalados como de mayor riesgo perinatals. 15, 16.

El desarrollo normal en los 6 casos en los cuales se demostró hemorragia subaracnoídea está de acuerdo con lo señalado por J. Volpe ${ }^{t}$ en cuanto al buen pronóstico de las hemorragias subaracnoídeag primarias. Es probable que ninguno de estos seis niños haya tenido hemorragia periventricular-intracerebral de importancia, casos en los cuales el pro nóstico en cuanto a vida y a secuelas es muy pobre $\mathbf{~}$. Falta en esta serie un estudio diagnóstico preciso con tomografía axial para precisar o descartar la existencia de hemorragias intracraneanas localizadas que pueden alcanzar frecuencias hasta de $43 \%$ entre recién nacidos con peso de nacimiento inferior a 1500 grs. como lo ha demostrado L. A. Papile y cols. ${ }^{19}$.

Como puede suponerse en un estudio de este tipo, no fue posible establecer relaciones causales precisas entre antecedentes o factores patológicos perinatales y niveles de desarrollo a los 2 a 4 años de edad. Pero, como puede verse en las Tablas 6 y 7 , entre los antecedentes de los niños con retraso moderado o profundo del desarrollo se apreció un relativo mayor número de casos con malformaciones congénitas diversas, hipoglicemia, policitemia, desnutrición fetal. y problemas sociales. No fue posibje incluir en el presente estudio a niños que hubieran sufrido situaciones hipóxicas perinatales graves. tanto por las limitaciones para cuantificar la hipoxia como por la insuficiencia de los datos anamnésticos con que los niños fueron referidos desde las respectivas maternidades. La inclusión en el seguimiento de niños con antecedentes de asfixia perinatal severa y prolongada habria cambiado probablemente los resultados.

La relación entre niveles de desarrollo de niños incluidus en este estudio y los niveles socioeconómicos de los respectivos grupos familiares mostró ser significativamente diferente entre los niveles socioeconómicos alto a medio y el nivel bajo. El $89.7 \%$ de los hijos de familias de nivel socioeconómico alto o medio tuvo desarrollo norrnal, mientras sílo el 
$52.6 \%$ de los niños provenientes de familias de nivel socioeconómico bajo tuvo test de desarrollo normal. La proporción de nínos con retrasos leves y moderados fue mayor en los niños del nivel socioeconómico bajo. Los tres casos con retraso profundo del desarrollo pertenecían también al grupo de nivel socioeconómico bajo. pero en ellos las causas probables del retraso incluyen la coexistencia de otros factores (Tabla N. ${ }^{\circ}$ ). La influencia del nivel socioeconómico del grupo familiar sobre los niveles de desarrollo de los niños a los 2 a 4 años puede explicarse por la interacción de diversos factores tales como el control prenatal. la atención del parto, nutrición materna y del lactante, controles de niño sano, higiene y estimulación psíquica y sensorial del lactante. Puede pensarse en base a estos resultados que niños con iquales complicaciones perinatales pueden mejorar su pronóstico si después del alta hospitalaria reciben mejores cuidados y mayor estimulación psicosensorial. Si se acepta esta conclusión se hace necesario reforzar la educación de los padres y poner en marcha programas controlados de estimulación para aquellos recién nacidos que se consideren de alto riesgo neurológico.

Los resultados de este estudio corresponden a la evaluación de niños considerados de alto riesgo neurológico que fueron atendidos en el Centro de Prematuros del Hospital L. Calvo Mackenna antes de la implementación de una Unidad de Cuidado In tensivo Neonatal en este Centro. La experiencia de centros extranjeros revela que al implementarse unidades de cuidado intensivo, el número de secuelas disminuye 3 a 20 . Será importante en nuestro medio evaluar el pronóstico de desarrollo de niños que sean atendidos en las diversas Unidades de Cuidado Intensivo Neonatal que se están poniendo en marcha a lo largo del pais como parte del Programa Nacional de Perinatología.

\section{RESUMEN}

Se evaluó el desarrollo de 77 niños considerados de "alto riesgo neurológico" en el período neonatal, y de 24 niños sin antecedentes de morbilidad perinatal. a la edad de 2 a 4 años. Se determinó el nivel de desarrollo aplicando la "Escala de Desarrollo Mental" de Griffiths y se expresaron los resultados en 4 niveles: normal, retraso leve, retraso moderado y retraso profundo.

En el grupo control el desarrollo fue normal en 20 niños $(83.3 \%)$, y 4 casos tenían retraso leve $(16.7 \%)$. En el grupo de "alto riesgo neurológico" hubo 55 casos con desarrollo normal (71.4\%) 10 casos con retraso leve ( $13 \%), 9$ casos con retraso moderado (11.7\%), y 3 casos con retraso profundo del desarrollo (3.9\%). La comparación del grupo de estudio con el grupo control no mostró diferencia significativa.

Los niveles de desarrollo de los niños con peso de nacimiento inferior a 1501 grs. (56 casos), con peso bajo para la edad gestacional ( 23 casos), o con anlecedentes de hipoglicemia neonatal ( 22 nin̄os), analizados por separado, tampoco difieren significativamente del grupo control. Se encontró sin embargo diferencia significativa entre los niveles de desarrollo alcanzados por niños pertenecientes a familias de distintos niveles socioeconómicos: los nin̄os de nivel sociceconómico medio $o$ alto tuvieron desarrollo normal en el $89.7 \%$ de los casos, mientras sólo el $52.6 \%$ de aquellos de nivel socioeconómico bajo tuvo test de desarrollo normal.

\section{REFERENCIAS}

${ }^{1}$ M. Ferreiro, J. P. Beca, R. Maler, S. Vaisman, Implementación de la atención neoaclal en Chile. Pedjatrín 19: 282, 1976.

2R. Griffiths. The Habilities of Young Children. Young \& Son Printen Lid., Cherd Sumerset, U. K., 1970,

${ }^{3}$ L. O. Lubchenco et al. Sequelae of Premature Birth. Am. J. Dis. Children 106: 101, 1963.

4L. O. Lubchenco, H. Band, A. L. Coldman, et al. Newbom Intensive Care and Long-tem Prognosis. Develop. Med. Chihd Neurol. 16: 421, 1974.

$5_{I}$. Bjerre. Neurological Inveatigation of 5 year old Children with Low Birthweight. Acta Paed. Scand. 64: 859, 1975.

6P. A. Davies, J. P. M. Tizard. Very Low Birthweight and Subsequent Veuralugical Defect. Develnp. Med. Child Neurol. 17: $3,1975$.

${ }^{T}$ K. E. Pape, F. J. Burcic, S. Ashby, P. M. Fitzhardinge. The Status at Two Years of Low Birthweithr Infanta born in 1974 with Birthweits of less than 1001 grs. J. Pediatr. 92: 253, 1978.

BL. O. Lubchenco et al. Long-term Follow-up Studies of Prema. turely bom Infante. If Inflnence of Birthweight and Gestational Age on Sequelae. J. Pediatr. 80: 509, 1972.

'P. A. Davies, A. L. Stewart. Low Birthweight Infanls: Neurological Scquelae and Latem Inteligence. Brit. Med. Bull. 31; 85. I975.

10A. Griffiths, G. M. Bryant. Assessment of Effects of Neonatal Hypoglicesmis. Arch. Dis, Childh. 46: 819, 1971.

11J. O. Commey. P. M. Fitzhardinge. Handicap in the Preterm Sonall for Gealational Age Infant. J. Pediatr. 94: 779. 1979.

${ }^{12} \mathrm{H}$. P. Chase et al. Intra-uterine Undernutrition and Brain Development. Pediatrics 47: 491, 1971.

${ }^{13}$ E. M. Widdowson, D. E. Grabb, R. D. C. Milner. Cellnlar Development of some Human Organs befose Birth. Anch. Dir. Chiluth. 47: 652, 1972.

14 M. Winick. Cellular Growth in Introuterine Malnutrition. Pediat. Clin. of N.A. 17: 69, 1970.

${ }^{15}$ C. M. Drillian. The Small for Date Infant: Ethiology and Prognosis. Pediat. Clin, of N.A. 17: 9, 1970.

${ }^{16} \mathrm{C}$. Amiel-Tison. Neurologic Problems in Perinatology. Clinice in Perinatology $L: 33,1974$.

17f. Volpe Neonalal lntracraneal Haemorrbage - Iatcogenic Etiology? N. Engl. J. Mad. 291: 43, 1974. 
19]. Volpe. Neonatal Periventricular Haemorthage: Pagt, present and future. J. Pediatr. 92: 693, 1978.

${ }^{19}$ L. A. Papile, J. Burstein, R. Burstein, H. Kaffler, Incidence and evolution of subependymal and intraventricular haemmorrhage:
A study of infants with birthweights less than 1500 ars. J. Pediatr. 92: 529, 1978.

${ }^{20}$ K. Rawlings, E. O. R. Reyrolds, Changing Prognosis for Infents of Very Low Birthweight. Lancet I: 316, 1971. 